\title{
Observation of doubly-periodic solutions of the nonlinear Schrödinger equation in optical fibers
}

\author{
Guillaume Vanderhaegen ${ }^{1}{ }^{*}$, Pascal Szriftgiser $^{1}$, Corentin Naveau ${ }^{1}$, Alexandre \\ Kudlinski $^{1}$, Matteo Conforti ${ }^{1}$, Stefano Trillo ${ }^{2}$, Nail AkhmedieV ${ }^{3}$, and Arnaud Mussot ${ }^{1,4}$ \\ ${ }^{1}$ Univ. Lille, CNRS, UMR 8523-PhLAM - Physique des Lasers Atomes et Molécules, F-59000 Lille, France \\ ${ }^{2}$ Department of Engineering, University of Ferrara, Via Saragat 1, 44122 Ferrara, Italy \\ ${ }^{3}$ Department of Theoretical Physics, Research School of Physics, The Australian National University, Canberra, ACT 2600, Australia \\ ${ }^{4}$ Institut Universitaire de France (IUF) \\ ${ }^{*}$ Corresponding author: guillaume.vanderhaegen@univ-lille.fr
}

Compiled May 12, 2020

We report the first experimental observation of doubly-periodic first-order solutions of the nonlinear Schrödinger equation in optical fibers. We confirm, experimentally, the existence of A and B type solutions. This is done by using the initial conditions that consists of a strong pump and two weak sidebands. The evolution of power and phase of the main spectral components is recorded using sophisticated heterodyne timedomain reflectometry. Another important part of our experiment is active loss compensation. With this setup, we reach a good agreement between theory and experiment. () 2020 Optical Society of America

http://dx.doi.org/10.1364/ao.XX.XXXXXX

The nonlinear Schrödinger equation (NLSE) describes the combined action of second order dispersion and cubic nonlinearity on a narrow-banded field envelope. It models wave evolution in many fields of physics describing basic nonlinear phenomena such as modulation instability (MI), which is at the origin of more complex scenarios such as rogue waves [1] or supercontinuum generation [2]. MI describes the initial stages of growth of a weak modulation at the expense of a strong continuous wave (CW) background and is ubiquitous in physics [3]. The amplification eventually saturates and is followed by a return to the initial state. Repeated cycles of growth and decay are referred to as Fermi-Pasta-Ulam recurrence [4], which has been widely investigated in hydrodynamics [5, 6] and optics [7-15]. In this context, the family of analytical solutions of the NLSE known as Akhmediev Breathers (AB) $[16,17]$ describes the nonlinear development of MI and is recognised to be a good prototype for rogue wave description [1]. These particular solutions of the NLSE are periodic in time and localised (hyperbolic) in space. They can be interpreted as the separatrices in an infinite-dimensional phase space $[8,16]$. As such, ABs account for a single stage of growth to the apex and the asymptotic return to the initial state, whereas they do not allow to predict the formation of multiple recurrences observed in recent experiments [6, 7, 10-15].

Approximate expressions for the location of the first maximal pulse compression point and for the spatial period as functions of the excited input signal parameters have been obtained recently by means of finite-gap theory and asymptotic expansions [18]. However, exact description of these parameters requires more general solutions. These expressions have been found by Akhmediev et al., in the form of first-order doubly periodic solutions [19]. The descriptive term 'first-order' means that the real and imaginary parts of each solution of this family satisfy a linear relationship, so that they describe a straight line in the complex plane at each propagation step (see [19] for details). The major properties of these solutions have been recently analysed in depth, and their Fourier coefficients that are important for many applications have been calculated analytically in [20]. Particular solutions of the NLSE on a finite background such as $\mathrm{AB}$, Peregrine soliton, and Kuznetsov-Ma soliton are special cases of this more general three-parameter family of solutions. They have been observed in several experimental works [21, 22].

First-order solutions, in turn, can be subdivided in two types, A or B, according to their location in the infinite-dimensional phase space with respect to the separatrix represented by the AB $[6,16]$. These analytical solutions have not been observed so far in any optical experiment due to the complexity of exciting them in realistic experimental conditions. It is a challenge to record both the phase and power evolution of the field and its Fourier components required for a detailed comparison between experimental data and the theory. Dissipation having a strong impact on the nonlinear stage of modulation instability [6], another issue to address is the linear losses of fiber.

In this Letter, we report the first observation of these doublyperiodic first-order solutions of the NLSE in an optical fiber. Remarkably, we demonstrate that they can be excited with good accuracy by simple three-wave initial conditions. The evolution of the power and phase along the fiber is recorded using a sophisticated heterodyne time-domain reflectometer (HOTDR) [12]. The optical losses inevitable in long fibers are actively compensated with a distributed Raman amplifier. These recordings are compared with the first two Fourier components of the doubly-periodic A and B type solutions found in [20].

Propagation of the light envelope $A(z, t)$ in the fiber is described by the NLSE, which, consistently with $[16,19,20]$, we 
conveniently write in the following dimensionless form

$$
i \frac{\partial A}{\partial z}+\frac{1}{2} \frac{\partial^{2} A}{\partial t^{2}}+|A|^{2} A=0,
$$

where $z$ is the distance along the fiber and $t$ is retarded time in the frame moving with the group velocity. From non-dimensional quantities, we obtain the real-world electric field $E$, distance $Z$ and retarded time $T$ as $E=A \sqrt{P}, Z=z /(\gamma P)$, and $T=t T_{0}$, where $T_{0}=\sqrt{\left|\beta_{2}\right| /(\gamma P)}$, and henceforth $P=490 \mathrm{~mW}, \beta_{2}=$ $-19 \times 10^{-27} \mathrm{~s}^{2} / \mathrm{m}$, and $\gamma=1.3 \times 10^{-3} \mathrm{~W}^{-1} \mathrm{~m}^{-1}$ are the total injected power, the anomalous group-velocity dispersion, and the nonlinear coefficient in our experiment.

As already mentioned, there are two qualitatively different types of doubly periodic solutions of Eq. (1). They differ by the value of phase shift of successive maxima along the $z$-axis. The solutions with all the maxima in phase are the B-type. The solutions with the alternating phases of the maxima are the A-type solutions. These two types of solutions are displayed in Fig. 1. The left panels show the B-type and the right panels show the Atype solutions. These analytical solutions have been computed
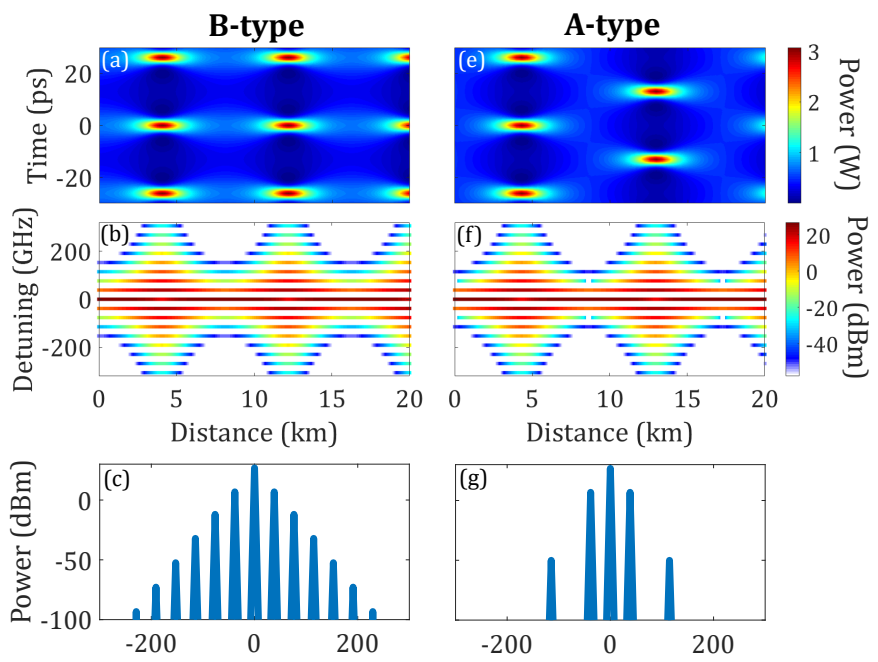

Frequency detuning (GHz)

Frequency detuning (GHz)
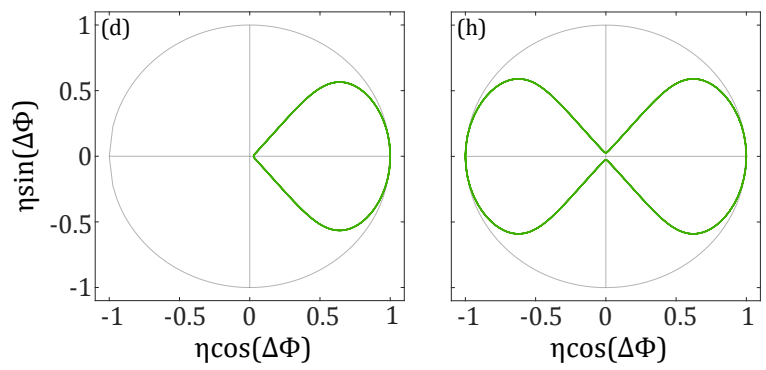

Fig. 1. Left hand side panels: B-type doubly periodic solution. Right hand side panels: A-type doubly periodic solution. (a), (e) Spatio-temporal evolution of the power. (b), (f) Longitudinal evolution of the modulus of the spectral components. (c), (g) Spectra of the solutions at the point $z=0$ (input of the fiber). (d), (h) Phase-plane representation of these solutions.

using the expressions reported in [20]. The spatio-temporal evolution of these doubly-periodic solutions are presented in Figs. 1 (a) and (e). The periodicity in the time domain is observed in the form of a train of pulses. The periodicity in the spatial domain appears as periodic wave evolution along the fiber. The latter is limited in the figure by slightly more than two recurrences. For the B-type solution, the maximum pulse compression points appear at the same temporal positions in each recurrence, while for the A-type solution they are shifted by half of the temporal period.

Figures 1 (b) and (f) show the power evolution of their spectral components. In each case, the central component that corresponds to the $\mathrm{CW}$ background serves as the source of power for the signal and idler waves and all higher-order sideband pairs generated through multiple four-wave mixing processes. Remarkably, all these processes are described by the exact solution of a single equation - NLSE. The process of energy transfer reverses after the maximal spectral expansion leading back to the initial spectrum after one period of evolution, thus, completing the FPU recurrence. The input spectra of both types of solutions are displayed in Figs. 1 (c) and (g). They are dramatically different: the B-type solution has a triangular-shaped spectrum at $z=0$, while the spectrum of the A-type solution contains only odd order sidebands. The even order sidebands are exactly zero. This suggests that A-type could be easily excited by means of truncated three-wave input, the third-order sidebands in Fig. 1 (g) being nearly $60 \mathrm{~dB}$ below the main ones. Conversely, for B-type the excitation via three waves appears to be more critical and should be tested.

Although, in principle, exact initial conditions can be created [23], but in reality, controlling more than three waves propagating in a fiber is difficult. In our experiments, we indeed used a three-wave input (the pump and the signal-idler pair). This is sufficient to excite both types of doubly-periodic solutions with reasonable accuracy, provided that the signal-idler pair is sufficiently weak. Indeed, as indicated by our extensive numerical simulations, weak input modulations guarantee that a higher fraction of total power remains confined in the main three frequencies along the fiber, thus reducing the impact of higher-order sidebands. In turn, too weak input modulations (for fixed pump power) result into too long recurrence distances (spatial periods). As a trade-off, in our experiment, the signal to pump ratio is set at $-20 \mathrm{~dB}$ (yet, typically more than one order of magnitude weaker than in previous experiments [12-14]). Given the experimental limitation in fiber length $\left(Z_{\max }=16.54\right.$ $\mathrm{km}$ ) and achievable power $(P \sim 0.5 \mathrm{~W}$ or $27 \mathrm{dBm})$, this is still sufficient to observe the second peak growth (expected around $Z \sim 12-13 \mathrm{~km}$, as shown in Fig. 1 (a),(b)), which is where A-type and B-type solutions differ for the temporal shift.

A convenient finite phase-space representation of the solutions can be given by reporting the associated trajectories in the phase-plane $(\eta \cos (\Delta \Phi), \eta \sin (\Delta \Phi))$, as displayed in Figs. 1 (d) and (h). Here, $\eta$ is the power of the first-order Fourier coefficient normalised to its maximum value and $\Delta \Phi$ is the phase difference between the zero (pump) and the first (signal) order Fourier coefficients of the solutions. The B-type solutions are located inside a separatrix ( $A B$, not shown), which means that two consecutive growth-decay cycles follow the same path in the right half of the phase plane. In contrast, the A-type solution are located outside the separatrix, which means that the two consecutive cycles are located in the opposite half planes. This leads to the $\pi$-shift of the maxima of the two cycles. In both cases suitable initial conditions are represented by the closest approach to the origin which yields the shallowest modulation (minimum of $\eta(z)$ ). As clear from Figs. 1 (d) and (h), this requires an input phase $\Delta \Phi=0$ for B-type, and $\Delta \Phi= \pm \frac{\pi}{2}$ for A-type, consistently with what already shown in previous experiments [12]. 
Importantly, we emphasize that those illustrated in Fig. 1 are two particular solutions of Eq. (1), out of a full family characterized by three arbitrary parameters, denoted $\alpha_{1}, \alpha_{2}, \alpha_{3}$ as in $[19,20]$. These parameters are non-zero roots of a fourthorder polynomial (Eqs. $(13,16)$ from $[19])$. They control the two (spatial and temporal) periods as well as the maximum amplitude. In our case, these three parameters are calculated numerically by matching, at the input of the fiber $(z=0)$, the amplitudes and the relative phases of the zero- and first-order Fourier components of the analytical solution with the amplitudes and relative phases of the three waves in the experiment. We obtain $\alpha_{1}=0.426, \alpha_{2}=0.728, \alpha_{3}=1.026$ for B-type, and $\alpha_{1,2}=0.565 \pm i 0.129, \alpha_{3}=0.980$ for A-type, which allows to uniquely specify the solution. Note that $\alpha_{1,2}$ are real for B-type and complex conjugate for A-type, while $\alpha_{3} \simeq 1$, as discussed in more detail in [20].

Our present experimental setup is similar to the one used in $[13,14]$. A simplified sketch of it is depicted in Fig. 2. Here, we

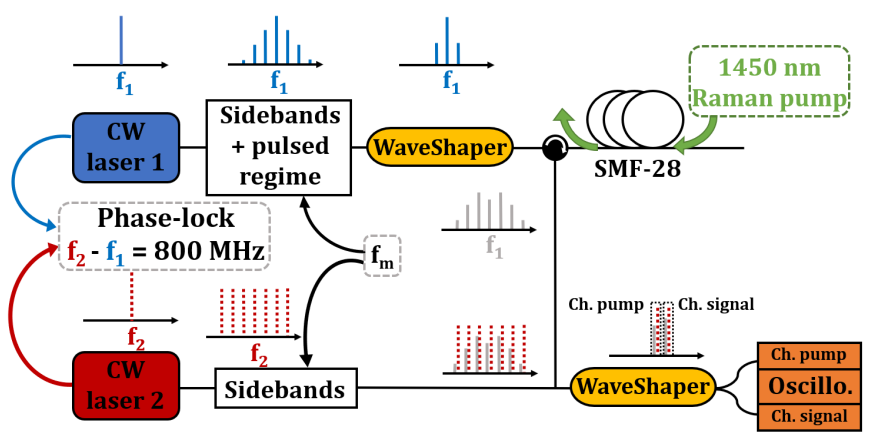

Fig. 2. Sketch of the experimental setup. $f_{1}$ and $f_{2}$ are the central frequencies of the main laser and the local oscillator respectively. The phase between them is locked to $f_{2}-f_{1}=800$ MHz. $f_{m}=38.2 \mathrm{GHz}$ is the modulation frequency.

provide the most important characteristics of the setup (for a more detailed description of this complex experimental apparatus, see [24]). A $1550 \mathrm{~nm} \mathrm{CW} \mathrm{laser} \mathrm{signal} \mathrm{is} \mathrm{weakly} \mathrm{modulated}$ with a phase modulator, creating a frequency comb with triangular profile. An intensity modulator further shapes the signal to form $50 \mathrm{~ns}$ square pulses with a $4.9 \mathrm{kHz}$ repetition rate in order to avoid stimulated Brillouin scattering [25]. The triangular frequency comb, at the output of the phase modulator is truncated to 3 waves with a Waveshaper. The latter also allows us to control the powers and phases of each of the remaining spectral components. These components are boosted by an erbium doped fiber amplifier and injected in a $16.54 \mathrm{~km}$ long SMF-28 optical fiber. The input pump power is $P_{P}=480 \mathrm{~mW}$ (total power $P \simeq 490 \mathrm{~mW}$ ), and the modulation frequency (sideband detuning) is $f_{m}=38.2 \mathrm{GHz}$, located close to the expected peak gain frequency $f_{\text {peak }}=\sqrt{2} /\left(2 \pi T_{0}\right) \simeq 41 \mathrm{GHz}$. The $z$-dependent relative phase of the pump, signal and idler waves, defined as $\Delta \Phi(z)=\phi_{P}(z)-\phi_{S}(z)$, is measured via HOTDR using the beating between the local oscillator and the Rayleigh backscattered signal from the waves propagating in the fiber.

Although the linear loss in SMF-28 is very low $(0.2 \mathrm{~dB} / \mathrm{km})$, the overall attenuation over the fiber length can strongly affect the dynamics. An active compensation of the losses is implemented using Raman amplification from a counter-propagating pump at $1450 \mathrm{~nm}$. It provides the maximum amplification at
1550 nm, i.e. 13.2 THz away from the Raman pump. Such compensation scheme keeps the total power nearly constant over the whole fiber length. Consequently, experiments are performed in a 'quasi-lossless' optical fiber. This, in turn, allows us to make a direct comparison between experimental measurements and the theory derived from the integrable (conservative) NLSE.

Experimental data for the B-type solutions are presented in Fig. 3 by solid lines. This solution is excited when the initial

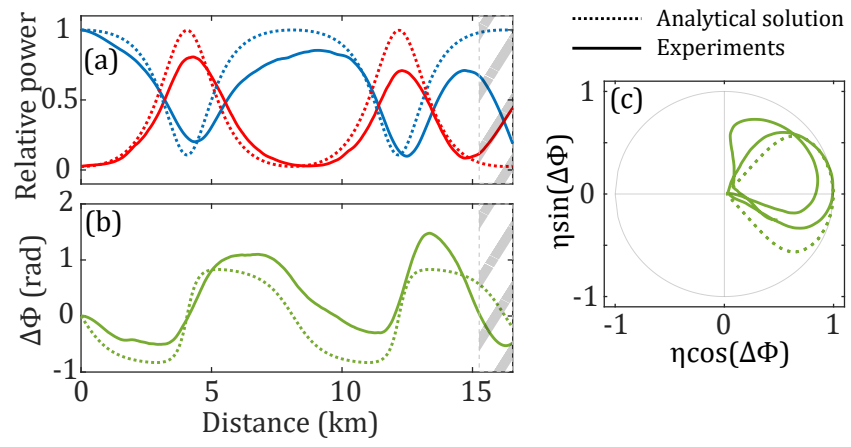

Fig. 3. Evolution, along the fiber, of (a) the pump (blue lines) and the signal (red lines) powers, (b) the relative phase. (c) Phase-plane representation. Dotted lines correspond to the B-type first-order solution of the NLSE and solid lines to the experimental data. Each component is normalized with respect to the maximum value of the analytical solution. The initial relative phase is set to $\Delta \Phi(z=0)=0$.

relative phase is $\Delta \Phi(z=0)=0$ [12]. The evolution of the pump (blue lines) and the signal (red lines) powers along the fiber are displayed in Fig. 3 (a). The pump power first decreases from its initial value and reaches a minimum at $Z=4.3 \mathrm{~km}$. At the same interval, the signal power increases starting from its initial value and reaches a maximum at the same point where the pump is minimal. The maximum level of the signal power at this point is about 30 times of the initial value. The signal power decreases afterwards to complete one full cycle of growth and decay. The second cycle of this periodic dynamics is observed in the range $Z \simeq 8-15 \mathrm{~km}$. Around $Z=15 \mathrm{~km}$ the pump power grows to its second maximum, which is around $70 \%$ of the initial power. Fig. 3 (b) shows the evolution of the relative phase along the fiber. These curves are also periodic. The two cycles correspond to those for power curves. The same dynamics in the phaseplane $(\eta \sin (\Delta \Phi), \eta \cos (\Delta \Phi))$ is shown in Fig. 3 (c) [8]. Here, $\eta=\frac{P_{S}}{\max \left(P_{S}\right)}$. The phase trajectory remains in the right half of the plane for both cycles of evolution. This behaviour is exactly what we expect for B-type solutions.

We compared these experimental results with the first-order solutions of the NLSE. The dotted lines in Fig. 3 correspond to the zero-order and the first-order Fourier coefficients of B-type solution shown in Fig. 1, calculated from Eqs. (13-16) in [20], and whose parameters were determined through the perfect match condition between the initial three central waves and the experimental three-wave input. The experimental data are in good agreement with this solution during the two cycles of evolution. In particular, the distance between minima of the pump is found in excellent agreement with the spatial period $Z_{\text {per }}=8.12 \mathrm{~km}$ of the solution, which is fixed (along with temporal period and amplitude) by the choice of the three arbitrary 
parameters discussed above. We attribute the discrepancies observed beyond the two cycles to the imperfect loss compensation by the back-propagating Raman pump. Indeed, the signal at the end of the fiber is amplified more than in the central part. The latter is the region where the lowest powers are reached [25]. Conditions for a quasi-transparent fiber are reached only along $15 \mathrm{~km}$ of propagation, whereas the signals in the last few kilometers of the fiber are over-amplified. This over-compensation leads to a higher total power in the last section, thus changing the parameters of the wave conversion process. Therefore this region (hatched in Figs. 3-4) should be omitted when comparing experimental data with the theory. Moreover, the gap of the envelope maxima between the experiments and the analytical solutions doesn't come from an incomplete compensation of the losses as the dynamic of the process (location of the extrema) fits with the theory. It is probably due to averaging process of the measurements and data processing.

Next, we set $\Delta \Phi(z=0)=-\frac{\pi}{2}$, in order to excite the A-type solution. The corresponding experimental recordings are plotted by solid lines in Fig. 4. As in the previous case, the evolution
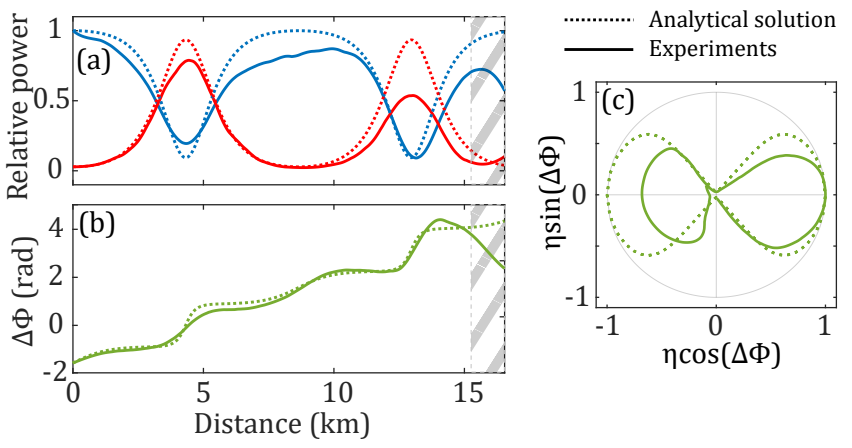

Fig. 4. Same as in Fig. 3 but for the A-type solution. Here $\Delta \Phi(z=0)=-\frac{\pi}{2}$.

along the fiber length of the pump (blue lines) and the signal (red lines) are presented in Fig. 4 (a). The power evolution curves are very similar to the B-type case. The location of the minima of the pump coincide pretty well with the location of the maxima of signal power, namely at $Z=4.4 \mathrm{~km}$ and $Z=13.1 \mathrm{~km}$. The signal power at the point of the maximal pulse compression is higher than its initial value by a factor of 30 . The last maximum of the pump power is $70 \%$ of its initial value.

Figure 4 (b) displays the evolution of the relative phase along the fiber. As can be seen, the relative phase does not return to the initial value, but rather increases monotonically. This means that the apex in the second cycle is phase-shifted by $\pi$ from the first one. The phase-plane projection is shown in Fig. 4 (c). The first growth-decay cycle occurs in the right half of the phase-plane while the second cycle occurs in the left half, which is the key feature of the A-type solution. Compared with previous case, this is equivalent to have crossed the separatrix.

These experimental results are also compared with the A-type solution of the NLSE. The dotted lines in Fig. 4 are calculated from the $0^{\text {th }}$ and $1^{\text {st }}$ order Fourier coefficients, according to Eqs. $(15,16,23,24)$ in $[20]$. The agreement between the analytical solution and the experimental data are reasonably good, especially for the relative phase evolution in Fig. 4 (b). The observed distance between maximal pulse compression points (maximum signal) fits within $1 \%$ with the theoretical value $Z_{\text {half }}=8.64 \mathrm{~km}$, which is equal to half period of the evolution (in this case the full period is the return of the phase to its initial value) [20]. Again, similar to the result in Fig. 3, the agreement is less accurate at the fiber end, due to an imperfect loss compensation.

In conclusion, we have reported the first observation of the doubly-periodic first-order solutions of the NLSE. This has been achieved in a loss-compensated optical fiber via HOTDR technique, which allowed us to record the evolutions of both powers and relative phases of the pump and signal waves. A direct comparison of the experimental data with analytical predictions is the result of these measurements. The agreement with the Fourier expansion [20] of the first-order doubly-periodic solutions [19] is good. We have shown also that these solutions are robust: while being composed of many spectral components, they can be excited by using simple initial conditions limited to the three main waves. We switched the excitation from the Atype to the B-type solutions by merely tuning the initial relative phase of the three waves. Our successful observation of these solutions of the NLSE suggests that they can serve as a useful theoretical background for nonlinear experiments in many other physical contexts. Our experiments also pave the way to the observation of more complex solutions, such as the rogue waves on the doubly-periodic background recently derived in [26].

Funding. Agence Nationale de la Recherche (Programme Investissements d'Avenir); Ministry of Higher Education and Research; Hauts de France Council; European Regional Development Fund (Photonics for Society P4S, FUHNKC, EXAT).

Disclosures. The authors declare no conflicts of interest.

\section{REFERENCES}

1. N. Akhmediev, J. Soto-Crespo, and A. Ankiewicz, Phys. Lett. A 373, 2137 (2009).

2. J. M. Dudley, G. Genty, F. Dias, B. Kibler, and N. Akhmediev, Opt. Express 17, 21497 (2009).

3. V. Zakharov and L. Ostrovsky, Phys. D 238, 540 (2009).

4. E. Fermi, J. Pasta, and S. Ulam, Collect Papers of Enrico Fermi, vol. 2 (Univ. Chicago Press, 1965).

5. B. M. Lake, H. C. Yuen, H. Rungaldier, and W. E. Ferguson, J. Fluid Mech. 83, 49-74 (1977).

6. O. Kimmoun, H. C. Hsu, H. Branger, M. S. Li, Y. Y. Chen, C. Kharif, M. Onorato, E. J. R. Kelleher, B. Kibler, N. Akhmediev, and A. Chabchoub, Sci. Reports 6, 28516 (2016).

7. G. Van Simaeys, P. Emplit, and M. Haelterman, Phys. Rev. Lett. 87, 033902 (2001).

8. S. Trillo and S. Wabnitz, Opt. Lett. 16, 986 (1991).

9. K. Hammani, B. Wetzel, B. Kibler, J. Fatome, C. Finot, G. Millot, N. Akhmediev, and J. M. Dudley, Opt. Lett. 36, 2140 (2011).

10. D. Pierangeli, M. Flammini, L. Zhang, G. Marcucci, A. Agranat, P. Grinevich, P. Santini, C. Conti, and E. DelRe, Phys. Rev. X 8, 041017 (2018).

11. A. Mussot, A. Kudlinski, M. Droques, P. Szriftgiser, and N. Akhmediev, Phys. Rev. X 4, 011054 (2014).

12. A. Mussot, C. Naveau, M. Conforti, A. Kudlinski, F. Copie, P. Szriftgiser, and S. Trillo, Nat. Photonics 12, 303 (2018).

13. C. Naveau, P. Szriftgiser, A. Kudlinski, M. Conforti, S. Trillo, and A. Mussot, Opt. Lett. 44, 763 (2019).

14. C. Naveau, P. Szriftgiser, A. Kudlinski, M. Conforti, S. Trillo, and A. Mussot, Opt. Lett. 44, 5426 (2019).

15. X. Hu, W. Chen, Y. Lu, Z. Yu, M. Chen, and Z. Meng, IEEE Photon. Tech. Lett. 30, 47 (2018).

16. N. N. Akhmediev and V. I. Korneev, Theor. Math. Phys. 69, 1089 (1986).

17. M. Erkintalo, G. Genty, B. Wetzel, and J. Dudley, Phys. Lett. A 375, 2029 (2011). 
18. P. Grinevich and P. Santini, Phys. Lett. A 382, 973 (2018).

19. N. N. Akhmediev, V. M. Eleonskii, and N. E. Kulagin, Theor. Math. Phys. 72, 809 (1987).

20. M. Conforti, A. Mussot, A. Kudlinski, S. Trillo, and N. Akhmediev, Phys. Rev. A 101, 023843 (2020).

21. B. Kibler, J. Fatome, C. Finot, G. Millot, F. Dias, G. Genty, N. Akhmediev, and J. M. Dudley, Nat. Phys. 6, 790 (2010).

22. B. Kibler, J. Fatome, C. Finot, G. Millot, G. Genty, B. Wetzel, N. Akhmediev, F. Dias, and J. M. Dudley, Sci. Reports 2, 463 (2012).

23. G. Xu, K. Hammani, A. Chabchoub, J. M. Dudley, B. Kibler, and C. Finot, Phys. Rev. E 99, 012207 (2019).

24. G. Vanderhaegen, P. Szriftgiser, A. Kudlinski, M. Conforti, S. Trillo, M. Droques, and A. Mussot, To be published Opt. Express (2020).

25. C. Headley and G. Agrawal, Raman Amplification in Fiber Optical Communication Systems (Academic Press, 2005).

26. J. Chen, D. E. Pelinovsky, and R. E. White, Phys. Rev. E 100, 052219 (2019). 


\section{FULL REFERENCES}

1. N. Akhmediev, J. Soto-Crespo, and A. Ankiewicz, "Extreme waves that appear from nowhere: On the nature of rogue waves," Phys. Lett. A 373, $2137-2145$ (2009).

2. J. M. Dudley, G. Genty, F. Dias, B. Kibler, and N. Akhmediev, "Modulation instability, akhmediev breathers and continuous wave supercontinuum generation," Opt. Express 17, 21497-21508 (2009).

3. V. Zakharov and L. Ostrovsky, "Modulation instability: The beginning," Phys. D 238, 540-548 (2009).

4. E. Fermi, J. Pasta, and S. Ulam, Collect Papers of Enrico Fermi, vol. 2 (Univ. Chicago Press, 1965).

5. B. M. Lake, H. C. Yuen, H. Rungaldier, and W. E. Ferguson, "Nonlinear deep-water waves: theory and experiment. part 2. evolution of a continuous wave train," J. Fluid Mech. 83, 49-74 (1977).

6. O. Kimmoun, H. C. Hsu, H. Branger, M. S. Li, Y. Y. Chen, C. Kharif, M. Onorato, E. J. R. Kelleher, B. Kibler, N. Akhmediev, and A. Chabchoub, "Modulation Instability and Phase-Shifted Fermi-Pasta-Ulam Recurrence," Sci. Reports 6, 28516 (2016).

7. G. Van Simaeys, P. Emplit, and M. Haelterman, "Experimental demonstration of the fermi-pasta-ulam recurrence in a modulationally unstable optical wave," Phys. Rev. Lett. 87, 033902 (2001).

8. S. Trillo and S. Wabnitz, "Dynamics of the nonlinear modulational instability in optical fibers," Opt. Lett. 16, 986-988 (1991).

9. K. Hammani, B. Wetzel, B. Kibler, J. Fatome, C. Finot, G. Millot, N. Akhmediev, and J. M. Dudley, "Spectral dynamics of modulation instability described using Akhmediev breather theory," Opt. Lett. 36, 2140-2142 (2011).

10. D. Pierangeli, M. Flammini, L. Zhang, G. Marcucci, A. Agranat, P. Grinevich, P. Santini, C. Conti, and E. DelRe, "Observation of FermiPasta-Ulam-Tsingou Recurrence and Its Exact Dynamics," Phys. Rev. X 8, 041017 (2018).

11. A. Mussot, A. Kudlinski, M. Droques, P. Szriftgiser, and N. Akhmediev, "Fermi-Pasta-Ulam Recurrence in Nonlinear Fiber Optics: The Role of Reversible and Irreversible Losses," Phys. Rev. X 4, 011054 (2014).

12. A. Mussot, C. Naveau, M. Conforti, A. Kudlinski, F. Copie, P. Szriftgiser, and S. Trillo, "Fibre multi-wave mixing combs reveal the broken symmetry of Fermi-Pasta-Ulam recurrence," Nat. Photonics 12, 303-308 (2018).

13. C. Naveau, P. Szriftgiser, A. Kudlinski, M. Conforti, S. Trillo, and A. Mussot, "Full-field characterization of breather dynamics over the whole length of an optical fiber," Opt. Lett. 44, 763-766 (2019).

14. C. Naveau, P. Szriftgiser, A. Kudlinski, M. Conforti, S. Trillo, and A. Mussot, "Experimental characterization of recurrences and separatrix crossing in modulational instability," Opt. Lett. 44, 5426-5429 (2019).

15. X. Hu, W. Chen, Y. Lu, Z. Yu, M. Chen, and Z. Meng, "Distributed measurement of fermi-pasta-ulam recurrence in optical fibers," IEEE Photon. Tech. Lett. 30, 47-50 (2018).

16. N. N. Akhmediev and V. I. Korneev, "Modulation instability and periodic solutions of the nonlinear schrödinger equation," Theor. Math. Phys. 69, 1089-1093 (1986).

17. M. Erkintalo, G. Genty, B. Wetzel, and J. Dudley, "Akhmediev breather evolution in optical fiber for realistic initial conditions," Phys. Lett. A 375, 2029-2034 (2011).

18. P. Grinevich and P. Santini, "The exact rogue wave recurrence in the NLS periodic setting via matched asymptotic expansions, for 1 and 2 unstable modes," Phys. Lett. A 382, 973-979 (2018).

19. N. N. Akhmediev, V. M. Eleonskii, and N. E. Kulagin, "Exact first-order solutions of the nonlinear Schrödinger equation," Theor. Math. Phys. 72, 809-818 (1987).

20. M. Conforti, A. Mussot, A. Kudlinski, S. Trillo, and N. Akhmediev, "Doubly periodic solutions of the focusing nonlinear schrödinger equation: Recurrence, period doubling, and amplification outside the conventional modulation-instability band," Phys. Rev. A 101, 023843 (2020).

21. B. Kibler, J. Fatome, C. Finot, G. Millot, F. Dias, G. Genty, N. Akhmediev, and J. M. Dudley, "The Peregrine soliton in nonlinear fibre optics," Nat. Phys. 6, 790-795 (2010).

22. B. Kibler, J. Fatome, C. Finot, G. Millot, G. Genty, B. Wetzel, N. Akhmediev, F. Dias, and J. M. Dudley, "Observation of Kuznetsov-Ma soliton dynamics in optical fibre," Sci. Reports 2, 463 (2012).

23. G. Xu, K. Hammani, A. Chabchoub, J. M. Dudley, B. Kibler, and C. Finot, "Phase evolution of peregrine-like breathers in optics and hydrodynamics," Phys. Rev. E 99, 012207 (2019).

24. G. Vanderhaegen, P. Szriftgiser, A. Kudlinski, M. Conforti, S. Trillo, M. Droques, and A. Mussot, "Observation of four fermi-pasta-ulamtsingou recurrences in an ultra-low-loss optical fiber," To be published Opt. Express (2020).

25. C. Headley and G. Agrawal, Raman Amplification in Fiber Optical Communication Systems (Academic Press, 2005)

26. J. Chen, D. E. Pelinovsky, and R. E. White, "Rogue waves on the doubleperiodic background in the focusing nonlinear schrödinger equation," Phys. Rev. E 100, 052219 (2019). 